\title{
Chemical Reaction Effects on Stretching/Shrinking Vertical Surface in Stagnation Point Flow
}

\author{
Mahani Ahmad Kardri, NorfifahBachok, Fadzilah Md. Ali, Norihan Md. Arifin
}

\begin{abstract}
The effects of chemical reaction on nanofluid vertical surface were considered on the flow of stagnation point. The numerical results were obtained by MATLAB to observe the impacts of the parameter of chemical reaction to the governing parameters. The results are graphically portrayed to observe the presence of dual solutions. The validation of the results is compared to the previous study and tends to give a favorable argument.
\end{abstract} flow.

Keywords: Chemical reaction, nanofluid, stagnation point

\section{INTRODUCTION}

Heat transfer in liquid, such as ethylene glycol, engine oil and water, has some limitations in the capabilities of heat properties that can cause limitations in many thermal applications. Most solids, specifically, metals, have high thermal conductivity. Therefore, fluid containing solid particles can substantially raise its conductivity. This is one of the important factors in industrial processing that will be highlighted for product enhancement. Inclusion of nanoparticles in the base liquid is a reliable method to enhance the heat conductivity. Enhancing heat transfer from electricity saving views, has been accepted widely in industrial sectors.

A discussion and observation on convective transport in nanofluids was reported by [1]. They added the possibility to use nanofluids in systems of nuclear. The efficiency in engineering sector, technology growth and electronic equipment can be influenced by the important role of mixed convection flow. Therefore, from that motivation, this study aims to fill the gap from the recent study that can contribute to the fundamental knowledge on mixed convection flow.

This study applies the Buongiornomodel that are well known among researchers in the same field. He highlights the important slip mechanisms in nanofluid as Brownian and thermophoresis diffusion. Not only interested in mixed convection flow, this study also includes the effect of chemical reaction, an effect that slowly gain attention among researchers. such as drying, evaporation and transferring energy, the heat and mass transfer with chemical reaction attracted

Revised Manuscript Received on September 14, 2019.

Mahani Ahmad Kardri, Faculty of Computer and Mathematical Sciences, Universiti Teknologi MARA, Perak Branch, Tapah Campus, Malaysia \& Institute for Mathematical Research, Universiti Putra Malaysia, Selangor, Malaysia.

Norfifah Bachok, Department of Mathematics and Institute for Mathematical Research, Universiti Putra Malaysia, Selangor, Malaysia.

Fadzilah Md. Ali, Department of Mathematics and Institute for Mathematical Research, Universiti Putra Malaysia, Selangor, Malaysia.

Norihan Md. Arifin, Department of Mathematics and Institute for Mathematical Research, Universiti Putra Malaysia, Selangor, Malaysia.
Because of the important functions in processing sector

researchers' interest. One of the researchers that show interest to examine such effect is [2]. He found that for the stretching/shrinking surface, there are dual solutions. Later, in [3] were continued the work done by [2] by focusing on cylinder. She observed that the unique solution exists for stretching case but not for shrinking case. She also makes a conclusion that consistent with the observation made by [2].

Recently, problem with mixed convection stagnation point flow in a nanofluid was considered on the surface of vertical stretching/shrinking [4]. Up to date, still no investigation has been made towards similar problem with the presence of chemical reaction. Therefore, this study emphasizes on that effect with thermophoresis and Brownian motion parameter are taken into consideration.

\section{FORMULATION OF THE PROBLEM}

As shown in Fig. 1, the laminar viscous problem and nanofluid's steady stagnation-point flow are considered.

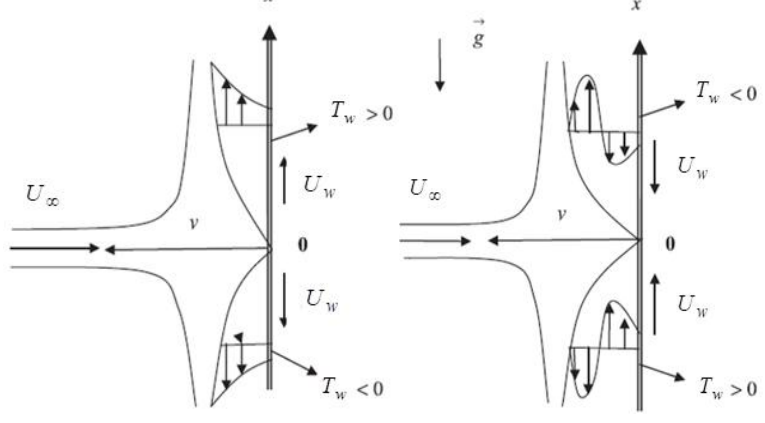

(a)

(b)

Fig. 1: Discussed model: (a) Assisting flow, (b) Opposing flow

The velocities involve are stretching/shrinking velocity $U_{w}(x)=a x$ and free stream velocity $U_{\infty}(x)=b x$ where constant $b>0$. The governing equation then will be express as [4]:

$$
\begin{aligned}
& \partial u / \partial x+\partial v / \partial y=0 \\
& u \partial u / \partial x+v \partial u / \partial y=U_{\infty} d U_{\infty} / \\
& +g / \rho_{f} \mid(1-C) \beta \rho_{f_{\infty}}\left(T-T_{\infty}\right)- \\
& u \partial T / \partial x+v \partial T / \partial y=\alpha_{n f} \partial^{2} T_{/} \\
& +\tau\left[D_{B} \partial T / \partial y \partial C / \partial y+D_{T} / T_{a}\right. \\
& u \partial C / \partial x+v \partial C / \partial y=D_{B} \partial^{2} C_{/} \\
& +D_{T} / T_{\infty} \partial^{2} T / \partial y^{2}-R\left(C-C_{\infty}\right.
\end{aligned}
$$




\section{CHEMICAL REACTION EFFECTS ON STRETCHING/SHRINKING VERTICAL SURFACE IN STAGNATION POINT FLOW}

subject to boundary conditions

$$
\begin{array}{llll}
u=U_{w}(x), & v=0, & T=T_{w}, & C=C_{w} \quad \text { at } y=0 \\
u \rightarrow U_{\infty}(x), & T \rightarrow T_{\infty}, & C \rightarrow C_{\infty} & \text { as } y \rightarrow \infty \quad(5)
\end{array}
$$

where the components of velocity denoted by $u$ and $v$. The viscosity of kinematic and the diffusivity of thermal represented by $v$ and $\alpha, \rho_{f}$ is the liquid density, $\tau=\left(\rho c_{p}\right)_{n p} /\left(\rho c_{p}\right)_{f}$ is the heat capacity ratio of nanoparticles, the Brownian and thermophoresis diffusion coefficient denoted by $D_{B}$ and $D_{T}$, and the constant pressure heat is $c_{p}$.

The similarity variables have been used to find the similarity solution for (1)-(4) with boundary condition (5)

$$
\begin{aligned}
& \eta=\left(U_{\infty} / v x\right)^{1 / 2} y, \\
& \psi=\left(U_{\infty} v x\right)^{1 / 2} f(\eta), \\
& \phi=\left(C-C_{\infty}\right) /\left(C_{w}-C_{\infty}\right), \\
& \theta=\left(T-T_{\infty}\right) /\left(T_{w}-T_{\infty}\right)
\end{aligned}
$$

where $u=\partial \psi / \partial y$ and $v=-\partial \psi / \partial x$ with stream function, $\psi$. The differential partial equations, (2)-(4) then was reduced to ordinary differential equations below

$$
\begin{aligned}
& f^{\prime \prime \prime}+f f^{\prime \prime}+1-f^{\prime 2}+\lambda \theta-N_{r} \phi=0 \\
& 1 / \operatorname{Pr} \theta^{\prime \prime}+f \theta^{\prime}+N b \phi^{\prime} \theta^{\prime}+N_{t} \theta^{\prime 2}=0 \\
& \phi^{\prime \prime}+L e f \phi^{\prime}+N t / N b \theta^{\prime \prime}-L e \beta \phi=0
\end{aligned}
$$

with new boundary conditions transformation from (5),

$$
\begin{aligned}
& f(0)=0, \quad f^{\prime}(0)=\varepsilon, \quad \theta(0)=1, \quad \phi(0)=1, \\
& f^{\prime}(\eta) \rightarrow 1, \quad \theta(\eta) \rightarrow 0, \quad \phi(\eta) \rightarrow 0 \quad \text { as } \quad \eta \rightarrow \infty
\end{aligned}
$$

where the stretching/shrinking parameter is $\varepsilon=a / b$, the chemical reaction parameter is $\beta=R / b$, the parameter of mixed convection is $\lambda=(1-C) \beta \rho_{f_{\infty}}\left(T-T_{\infty}\right) g / \rho_{f} b^{2} x$, the parameter of buoyancy ratio is $N_{r}=\left(\rho_{p}-\rho_{f_{\infty}}\right)\left(C-C_{w}\right) g / \rho_{f} b^{2} x$ and Reynolds number is $\operatorname{Re}_{x}=U_{\infty} x / v$. Meanwhile, the number of Prandtl and Lewis, Prand Le, the parameter of Brownian motion and thermophoresis, $\mathrm{Nb}$ and $\mathrm{Nt}$, are defined as

$$
\begin{aligned}
& \operatorname{Pr}=v / \alpha, \quad L e=v / D_{B}, \quad N b=(\rho c)_{p} D_{B}\left(C_{w}-C_{\infty}\right) /(\rho c)_{f} v \\
& N t=(\rho c)_{p} D_{T}\left(T_{w}-T_{\infty}\right) /(\rho c)_{f} T_{\infty} v
\end{aligned}
$$

In this research, there are several physical interests that are the coefficient of skin friction and number of Nusselt and Sherwood,

$$
\begin{aligned}
& C_{f}=\tau_{w} / \rho_{f} U^{2}, \\
& N u_{x}=x q_{w} / k\left(T_{w}-T_{\infty}\right), \\
& S h_{x}=x q_{m} / D_{B}\left(C_{w}-C_{\infty}\right), \\
& \text { Then, obtained (13) from (6), } \\
& C_{f}(\operatorname{Re})_{x}^{1 / 2}=f^{\prime \prime}(0), \\
& N u_{x}(\operatorname{Re})_{x}^{-1 / 2}=-\theta^{\prime}(0), \\
& S h_{x}(\operatorname{Re})_{x}{ }^{-1 / 2}=-\phi^{\prime}(0)
\end{aligned}
$$$$
\tau_{w}=\mu(\partial u / \partial y)_{y=0}
$$$$
q_{m}=-D_{B}(\partial C / \partial y)_{y=0}
$$

\section{RESULTS AND DISCUSSION}

This study focuses on the similar problem by [4] with chemical reaction effects. The reduced ordinary differential$$
q_{w}=-k(\partial T / \partial y)_{y=0}
$$

equations from partial differential equations were then solved numerically using similarity transformation technique by MATLAB, repeating the technique for solving PDE in [5].

Fig. 2-4 show the variation of $f^{\prime \prime}(0),-\theta^{\prime}(0)$ and $-\phi^{\prime}(0)$ with stretching/shrinking parameter, $\varepsilon$ and mixed convection parameter, $\lambda$. Clearly from the figures, the highest range of solution shown is for $\lambda=0.3$ and the smallest range of solution is $\lambda=-0.3$. As the value of $\lambda$ rising, the widest range of solution can be obtained. The critical values of $\varepsilon_{c}$ are shown in Fig. 2-4 for $\lambda=-0.3,0,0.3$. Unique solution exists for stretching sheet while dual solution exists for shrinking sheet.

Notice the variation of $f^{\prime \prime}(0),-\theta^{\prime}(0)$ and $-\phi^{\prime}(0)$ with stretching/shrinking parameter, $\varepsilon$ and chemical reaction parameter, $\beta$, from Fig. 5-7. As seen in those figures, unique solution exists for stretching sheet and dual solution exists for shrinking sheet at certain value of $\varepsilon$, similar with results from Fig. 2-4. The highest range of solution can be obtained at $\beta=0.5$ and the smallest range of solution at $\beta=0$. Unexpected results obtain from $\beta=1$ because it gives the result in between.

Table 1 listed the result comparison between previous results by [4] and present results. The results give a favorable agreement and prove that the present results is reliable.

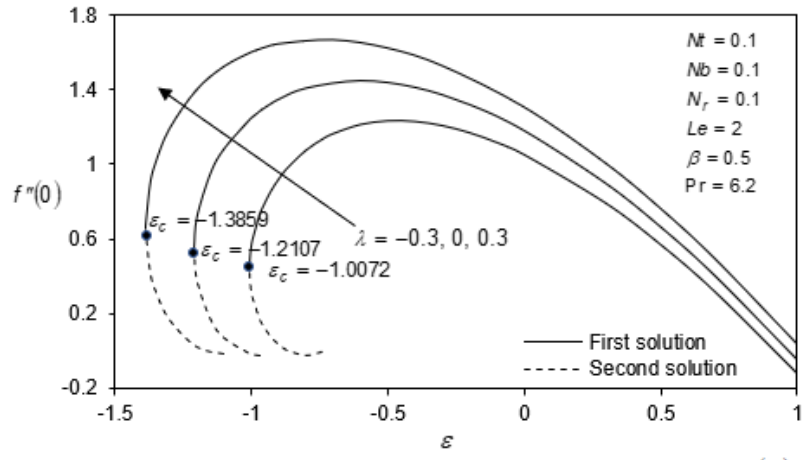

Fig. 2: Variation of the skin friction coefficient $f^{\prime \prime}(0)$ with $\varepsilon$ and $\lambda$

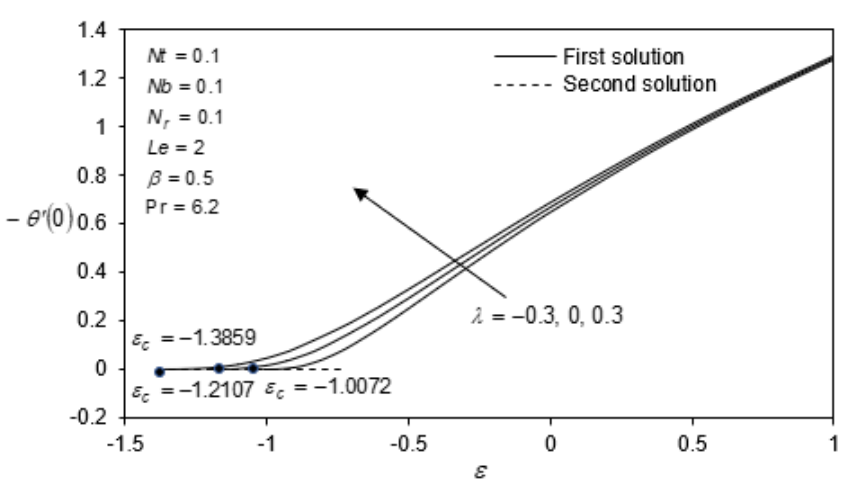

Fig. 3: Variation of the local Nusselt number $-\theta^{\prime}(0)$ with $\varepsilon$ and $\lambda$ 


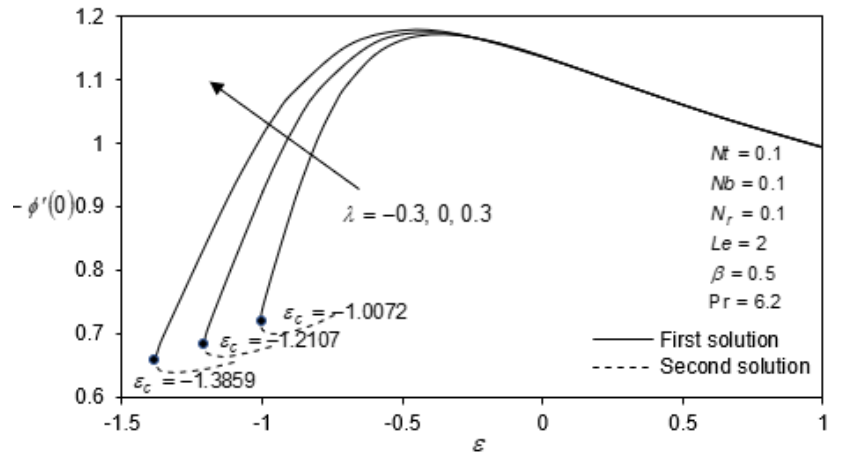

Fig. 4: Variation of the local Sherwood number $-\phi^{\prime}(0)$ with $\varepsilon$ and $\lambda$

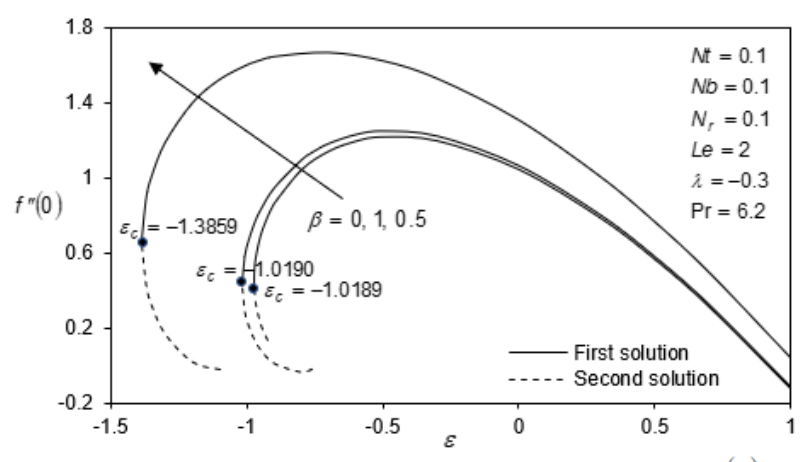

Fig. 5: Variation of the skin friction coefficient $f^{\prime \prime}(0)$ with $\varepsilon$ and $\beta$

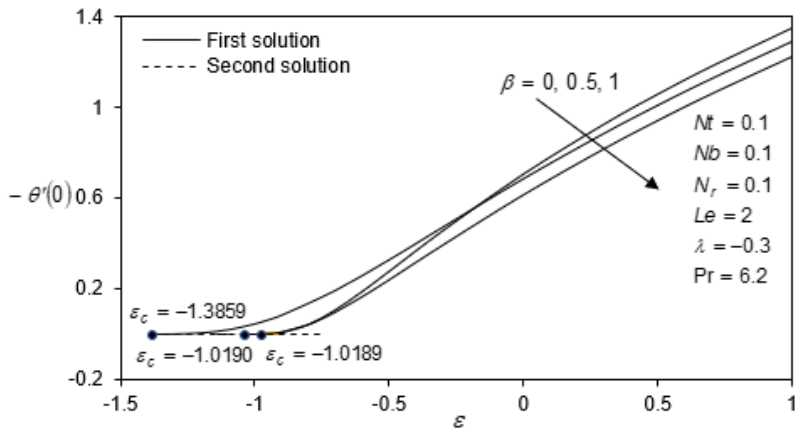

Fig. 6: Variation of the local Nusselt number $-\theta^{\prime}(0)$ with $\varepsilon$ and $\beta$

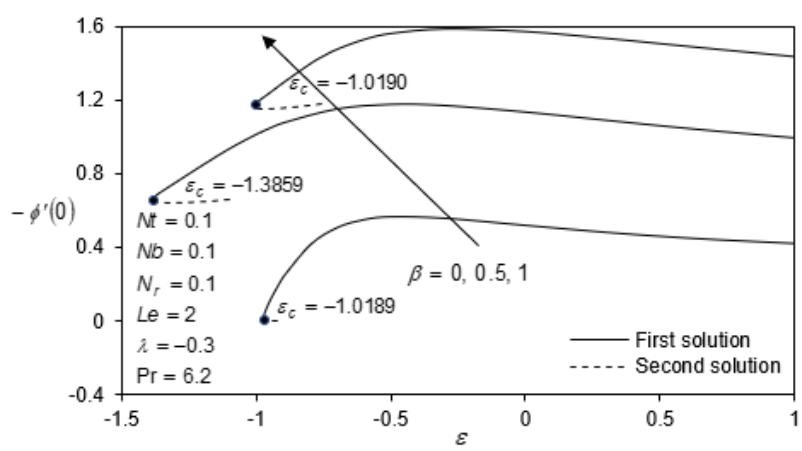

Fig. 7: Variation of the local Sherwood number $-\phi^{\prime}(0)$ with $\varepsilon$ and $\beta$

Table 1: Results comparison for the values of $f^{\prime \prime}(0),-\theta^{\prime}(0)$ and $-\phi^{\prime}(0)$ when $\operatorname{Pr}=7, N t=0.1, N b=0.1, N_{r}=0$ and $\varepsilon=-1$ for various values of $\lambda$

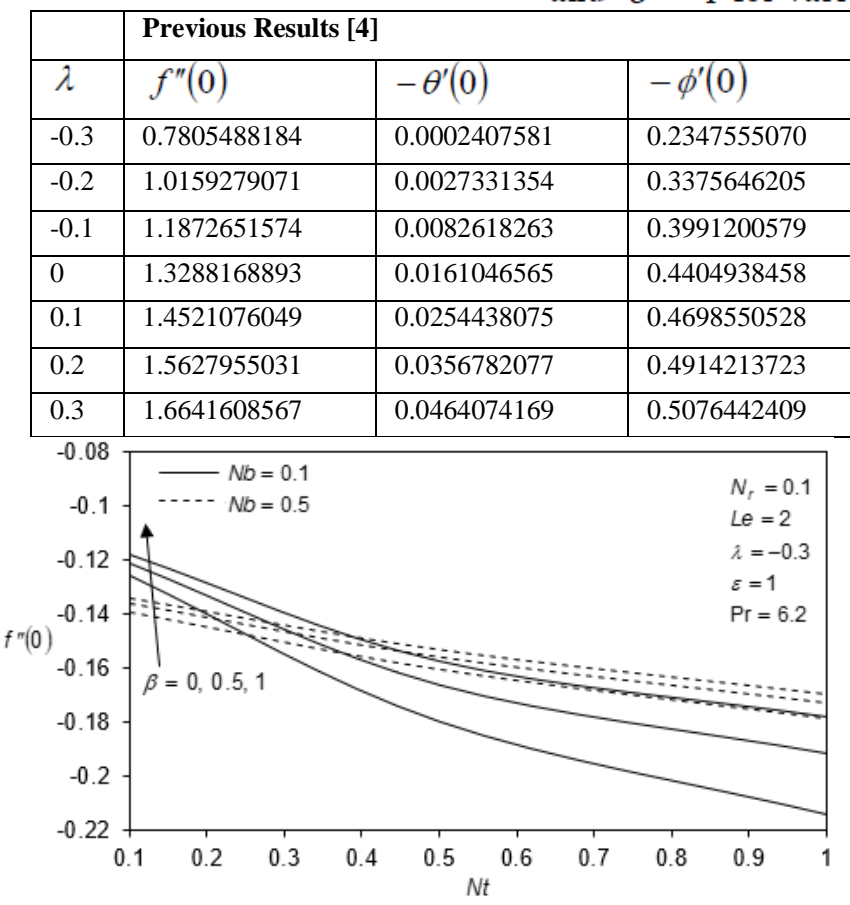

Fig. 8: Variation of the skin friction coefficient $f^{\prime \prime}(0)$ with $N t, N b$ and $\beta$

\begin{tabular}{|l|l|l|}
\hline \multicolumn{3}{|l|}{ Display Results } \\
\hline$f^{\prime \prime}(0)$ & $-\theta^{\prime}(0)$ & $-\phi^{\prime}(0)$ \\
\hline 0.7805487972 & 0.0002407622 & 0.2347254965 \\
\hline 1.0159278832 & 0.0027331453 & 0.3375646061 \\
\hline 1.1872651347 & 0.0082618380 & 0.3991200433 \\
\hline 1.3288168824 & 0.0161046606 & 0.4404938381 \\
\hline 1.4521075969 & 0.0254438122 & 0.4698550444 \\
\hline 1.5627954937 & 0.0356782124 & 0.4914213638 \\
\hline 1.6641608465 & 0.0464074223 & 0.5076442304 \\
\hline
\end{tabular}

Fig. 9: Variation of the local Nusselt number $-\theta^{\prime}(0)$ with $\mathrm{Nt}, \mathrm{Nb}$ and $\beta$ 


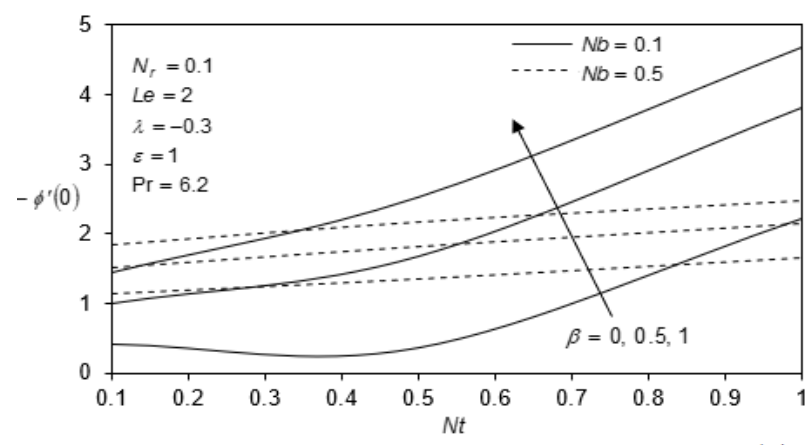

Fig. 10: Variation of the local Sherwood number $-\phi^{\prime}(0)$ with $N t, N b$ and $\beta$

Fig. 8-10 explain the results according to $N b, N t$ and $\beta$ for variation of $f^{\prime \prime}(0),-\theta^{\prime}(0)$ and $-\phi^{\prime}(0)$. Increases $N t$ is also to increase the $-\phi^{\prime}(0)$ as well, but to decrease the $f^{\prime \prime}(0)$ and $-\theta^{\prime}(0)$. The parameter of Brownian motion at $N b=0.1$ gives higher results compared to $N b=0.5$ except for $f^{\prime \prime}(0)$. Increases value of $\beta$ produced increases of $f^{\prime \prime}(0)$ and $-\phi^{\prime}(0)$ but decreases the value of $-\theta^{\prime}(0)$.

Fig. 11-13 depict the variation of $f^{\prime \prime}(0),-\theta^{\prime}(0)$ and $-\phi^{\prime}(0)$ with certain value of $\beta$ and $N t$ and the value of $N b$ varies. Increases the parameter of chemical reaction is to increase the $f^{\prime \prime}(0)$ and $-\phi^{\prime}(0)$. This phenomena repeating the results obtain from Fig. 7-9. The Nusselt number will decrease by increasing the value of $N b$. The parameter of thermophoresis at $N t=0.5$ tends to give higher range of solution than $N t=0.1$, as can be seen in Fig. 11 and Fig. 13 for $f^{\prime \prime}(0)$ and $-\phi^{\prime}(0)$.

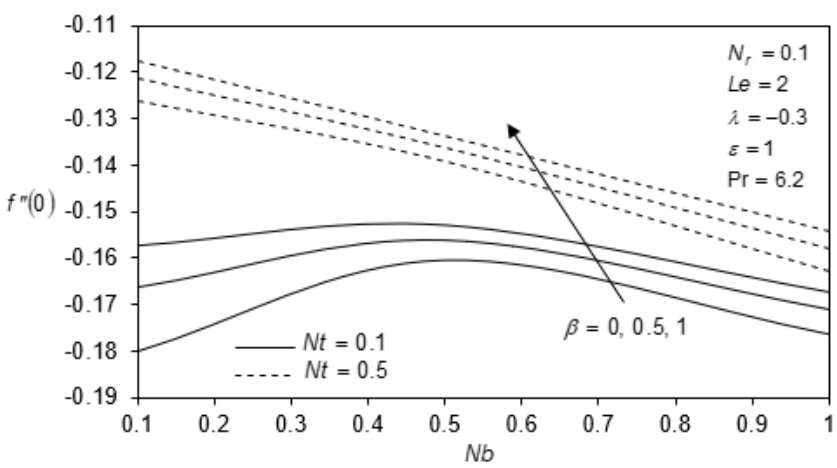

Fig. 11: Variation of the skin friction coefficient $f^{\prime \prime}(0)$ with $N b, N t$ and $\beta$

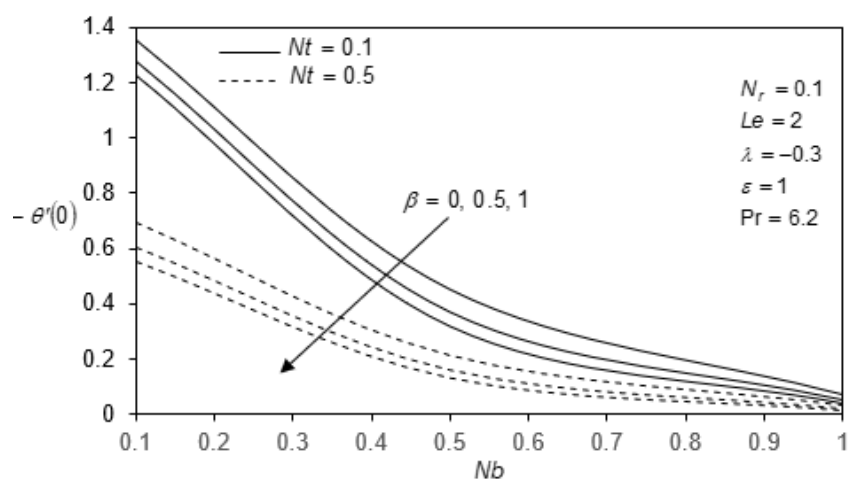

Fig. 12: Variation of the local Nusselt number $-\theta^{\prime}(0)$ with $N b, N t$ and $\beta$
Fig. 14-16 show the profiles of velocity, temperature and concentration on mixed convection parameter at $\lambda=-0.3$ and $\lambda=0$. It is found that, opposed to the other, the second solution has higher boundary layer thickness. Increases the value of $\lambda$ is to increase the first solution and decrease the velocity profile for second solution (see Fig. 14) but different concentration profile circumstances have occurred (see Fig. 16).

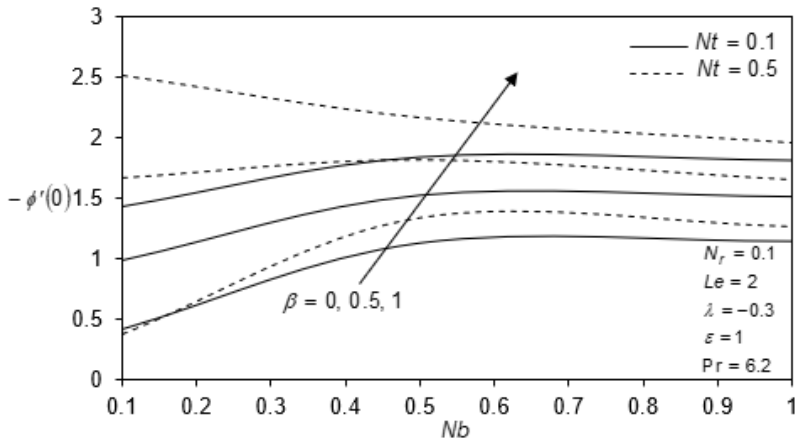

Fig. 13: Variation of the local Sherwood number $-\phi^{\prime}(0)$ with $N b, N t$ and $\beta$

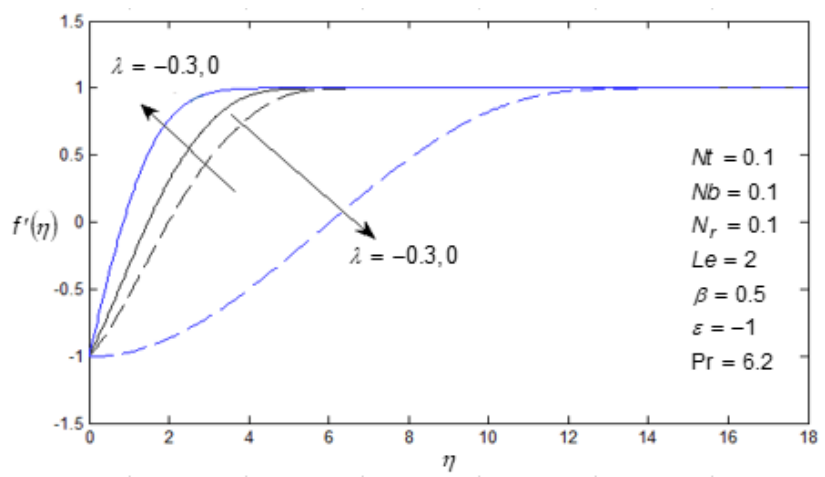

Fig. 14: Velocity profiles $f^{\prime}(\eta)$ for $\lambda=-0.3$ and $\lambda=0$

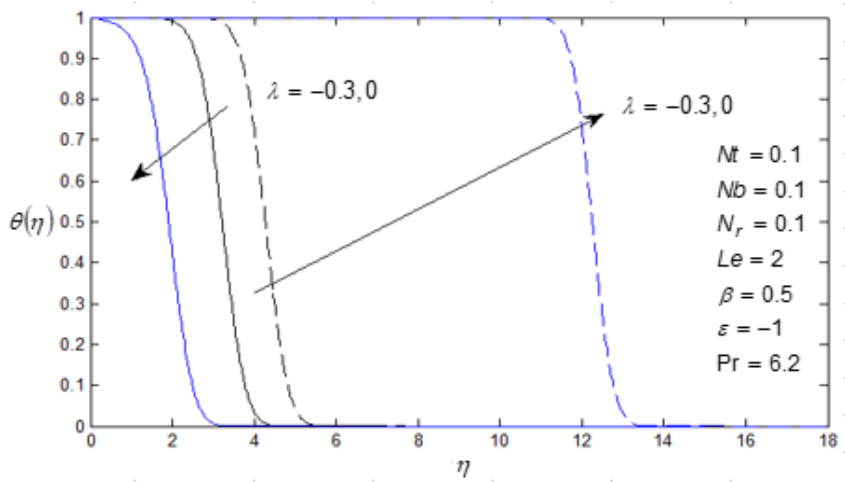

Fig. 15: Temperature profiles $\theta(\eta)$ for $\lambda=-0.3$ and $\lambda=0$ 


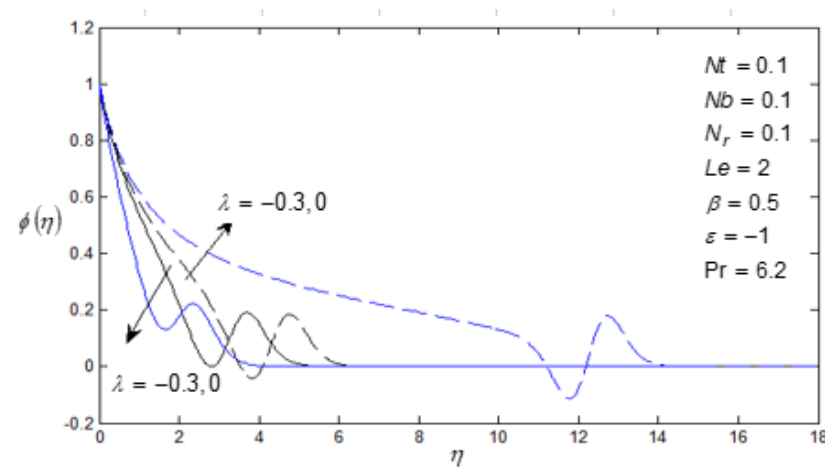

Fig. 16: Concentration profiles $\phi(\eta)$ for $\lambda=-0.3$ and $\lambda=0$

\section{CONCLUSION}

The chemical reaction effects were observed on a stretching/shrinking vertical surface in 2-D stagnation point flow. Three conclusions can be drawn,

- $\quad$ existence for the solutions of unique and dual on the case of stretching and shrinking, respectively,

- increases the parameter $\lambda$ is to increase the range of $f^{\prime \prime}(0),-\theta^{\prime}(0)$ and $-\phi^{\prime}(0)$, where increases of the reduced $f^{\prime \prime}(0)$, may leads to increase the rate of heat at surface,

- the chemical reaction effects are really high on $f^{\prime \prime}(0)$ and $-\phi^{\prime}(0)$.

\section{ACKNOWLEDGMENT}

The authors were grateful for funding received from Universiti Putra Malaysia and UniversitiTeknologi MARA. The comments from anonymous reviewers are highly appreciated.

\section{REFERENCES}

1 J. Buongiorno and W. Hu, "Nanofluid coolants for advanced nuclear power plants," Proceedings of ICAPP, 5(5705), 2005, pp. 15-19.

2 K. Bhattacharyya, "Dual solutions in boundary layer stagnation-point flow and mass transfer with chemical reaction past a stretching/shrinking sheet," International Communications in Heat and Mass Transfer, 38(7), 2011, pp. 917-922.

3 N. Najib, N. Bachok, N. M. Arifin, and A. Ishak, "Stagnation point flow and mass transfer with chemical reaction past a stretching/shrinking cylinder," Scientific Reports, 4, 2014, pp. 1-7.

4 N. A. Othman, N. A. Yacob, N. Bachok, A. Ishak, and I. Pop, "Mixed convection boundary-layer stagnation point flow past a vertical stretching/shrinking surface in a nanofluid," Applied Thermal Engineering, 115, 2017, pp. 1412-1417.

5 M. A. Kardri, N. Bachok, N. M. Arifin, and F. M. Ali, "Heat transfer and axisymmetric stagnation point flow due to a shrinking vertical plate in a nanofluid with slip effects," In Journal of Physics: Conference Series, 1132(1), 2018, p. 012026.

\section{AUTHORS PROFILE}

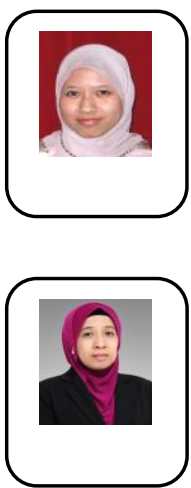

Mahani Ahmad Kardri is a senior lecturer in Mathematical Sciences Department, Faculty of Computer and Mathematical Sciences, UniversitiTeknologi MARA Perak Branch, Tapah Campus. She holds a Bachelor's Degree and Masters in Mathematics. Her research interests are Mathematics specialize in Fluid Dynamics.

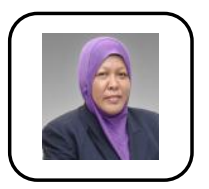

FadzilahMd Ali is a senior lecturer in Mathematics Department, Faculty of Science, Universiti Putra Malaysia. She holds a Bachelor's Degree, Masters and $\mathrm{PhD}$ in Mathematics. Her research interests are in Fluid Dynamics and Industrial Mathematics.

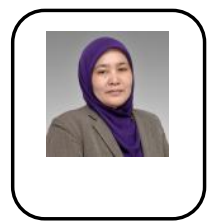

NorihanMdArifin is a Professor in Mathematics Department, Faculty of Science, Universiti Putra Malaysia. She holds a Bachelor's Degree, Masters and $\mathrm{PhD}$ in Mathematics. Her research interests are in Fluid Dynamics and Industrial Mathematics. 